Proc. Indian Acad. Sci. (Chem. Sci.), Vol. 96, No. 6, April 1986, pp. 559-564.

(C) Printed in India.

\title{
Low temperature properties of glasses-unsolved problems
}

\author{
A K RAYCHAUDHURI \\ Department of Physics, Indian Institute of Science, Bangalore 560012, India
}

\begin{abstract}
Glasses show very interesting behaviour weil below the glass transition temperature. Inspite of various experimental observations, even simple quantitative explanations relating these relaxation phenomena to structural properties are absent. In this paper we have tried to point out a phenomenological approach to this problem by identifying certain parameters which we think can be used to characterize these relaxations.
\end{abstract}

Keywords. Glasses; relaxation; tunnelling model.

\section{Introduction}

Below the glass transition temperaturc glasses show very interesting behaviour. These have been studied by various experimental methods. However, till today a microscopic understanding of these low temperature properties has not been obtained. A reason for this may be the lack of any attempt for a structure-property correlation. It is true that most of these properties of glasses are universal and arise from its disorder structure. Nevertheless, the details for each material will be different. This is because most of this low temperature behaviour arises from low energy structural rearrangements occurring most probably in the scale of nearest or next nearest neighbour. Thus the details of these structural rearrangement should be strongly dominated by short range structural order. In this paper we identify what we think are the outstanding problems in glasses below $T_{g}$. Due to size limitations our discussions are not going to be extensive, where appropriate we will refer to existing references.

\section{The problem of relaxation}

Most of the observed properties in glasses below $T_{\theta}$ have their origins in relaxation of some type. So the crucial issue here is the existence of particle motion in a localized scale and over a time scale which has a wide distribution. This particle motion is in addition to the already existing lattice vibrations which occur at a relatively faster rate. A good review of particle motion in glasses has been given by Rao and Parthasarathy (1985).

To show that relaxations in glasses never stop even at temperatures much lower than $1 \mathrm{~K}$ we refer to figure 1 . This figure shows internal friction $\left(Q^{-1}\right)$ in vitreous silica in a temperature range $0.01 \mathrm{~K}$ to $1000 \mathrm{~K}$. At very high temperature, the rise in internal friction is due to glass transition (henceforth called $\alpha$-relaxation) which is marked by large scale structural change. At low temperature the peak in $Q^{-1}$ is generally called $\beta$ relaxation. Below this peak $Q^{-1}$ remains flat for a while and then decreases only at temperatures below $0.1 \mathrm{~K}$. This behaviour is related to the existence of very low frequency excitations which we will refer to as two level system (TLS) relaxation (the 


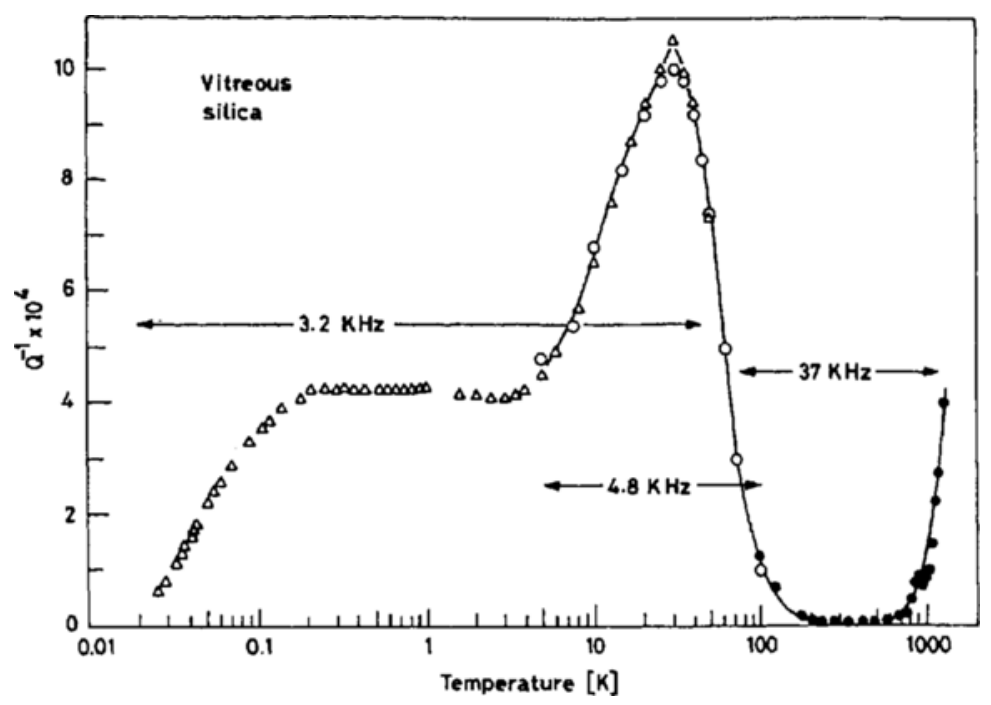

Figure 1. Internal friction $\left(Q^{-1}\right)$ of vitreous silica from $T=0.02 \mathrm{~K}$ to $T=1000 \mathrm{~K}$ ( $\Delta$ Raychaudhuri and Hunklinger 1984; $O$ Scott and MacGrone 1970; $\odot$ Silvertsen 1953).

meaning of which will become apparent shortly). $\alpha, \beta$ and TLs relaxations signify three different types of relaxation mechanisms. While $\alpha$ relaxation involves structural changes (unlocking of liquid-like defects as $T$ increases througk $T_{g}$ ) involving higher energy, $\beta$ relaxation involves localized structural changes. But both of them are classical thermally activated crossing of barriers although not of the pure Arrhenius type. TLS relaxation also involves localized structural changes, probably of the same type as in $\beta$ relaxation but the mechanism of relaxation is quantum mechanical in origin.

These relaxation mechanisms may themselves be interrelated. But nothing can be said at this moment of what the interrelation is. The key problem in the physics of glass is relaxation and the key question is what is relaxing. The problem, as pointed out earlier, is one of structure property correlation. What we mean by structure-property correlation may be qualified as follows. First, it is necessary to identify the parameters which may be said to represent a quantitative measure of the relaxation process. Second, these parameters should be linked with some bulk properties of the glassy system. These bulk properties now need be linked to actual structures through a structural model. It is from this model, we hope, that a microscopic understanding of these relaxation mechanisms will emerge. The task now is two-fold. First, it is necessary to identify on a semi-empirical basis the correlation of relaxation with structural parameters and second, to actually invent simple models which will explain the main aspects of these semi-empirical correlations. Unfortunately, such an approach is absent in this problem.

In the following, we will discuss briefly some important features of $\beta$ and TLS relaxations and will try to point out what are the important relaxational parameters.

\section{3. $\beta$-relaxation}

The phenomenon of $\beta$ relaxation is studied mainly by mechanical experiments (Hunklinger and Arnold 1976) or dielectric experiments (Johari 1976). In mechanical 
experiments ultrasonic frequencies were generally used earlier. But measurements in insulating glasses at audio frequencies have also been done in the past. Recently $\beta$ relaxation in metallic glasses has also been studied extensively by using audiofrequency techniques (Barmatz and Chen 1974; Raychaudhuri 1986).

In figure 2 we show ultrasonic attenuation in a number of glasses at low temperatures at a frequency of $20 \mathrm{MHz}$. In table 1, we list these materials, their glass transition temperatures $\left(T_{g}\right)$ and the temperature $T_{m}$ where the maximum occurs. It can be seen that $T_{m} / T_{g}$ shows extremely wide variation from 0.03 (for $\mathrm{SiO}_{2}$ ) to 0.27 (for $\mathrm{GeS}_{2}$ ). Of these the case of polymethyl methyl acrylate (PMMA) and polyethyl methyl acrylate (PEMA) is particularly interesting. Substitution of a methyl group by the heavier ethyl group shifts $T_{m} / T_{g}$ by a factor of 5 . This shift is due most probably to the increase in activation energy of relaxation.

In ultrasonic or mechanical experiments it is difficult to obtain data over a large frequency range. Generally, the frequency here is kept fixed and the temperature is varied. In case of dielectric relaxation, however, it is easier to obtain data in the frequency domain. Studies of relaxation by the dielectric method in a number of glass forming systems have been made (Johari 1976). From all these studies it is obvious that the $\beta$ relaxation peak has a large width. The first attempt to fit the experimental ultrasonic data was made by Hunklinger. He used a distribution of barrier heights $P(V)$

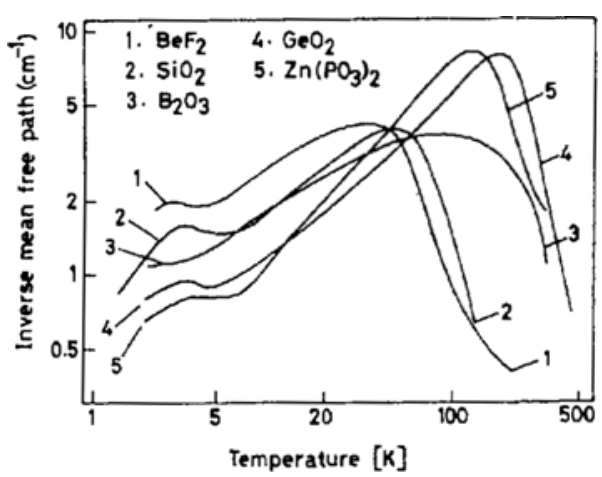

Figure 2. Ultrasonic attenuation in various glasses at low temperature at $20 \mathrm{MHz}$ (Hunklinger and Arnold 1976).

Table 1. Ultrasonic $\beta$-relaxation peaks in glasses (at $20 \mathrm{MHz}$ )

\begin{tabular}{lcccccccc}
\hline & \multicolumn{8}{c}{ Material } \\
\cline { 2 - 8 } & $\mathrm{GeS}_{2}$ & $\mathrm{GeS}_{4}$ & PMMA & PEMA & $\mathrm{SiO}_{2}$ & $\mathrm{GeO}_{2}$ & $\mathrm{BeF}_{2}$ & $\mathrm{~B}_{2} \mathrm{O}_{3}$ \\
\hline$T_{\theta}$ & $750 \mathrm{~K}$ & $625 \mathrm{~K}$ & $374 \mathrm{~K}$ & $338 \mathrm{~K}$ & $1475 \mathrm{~K}$ & $853 \mathrm{~K}$ & $623 \mathrm{~K}$ & $525 \mathrm{~K}$ \\
$T_{m}$ & $200 \mathrm{~K}$ & $150 \mathrm{~K}$ & $15 \mathrm{~K}$ & $70 \mathrm{~K}$ & $50 \mathrm{~K}$ & $170 \mathrm{~K}$ & $30 \mathrm{~K}$ & $75 \mathrm{~K}$ \\
$T_{m / T} / T_{0}$ & 0.27 & 0.24 & 0.04 & 0.21 & 0.03 & 0.20 & 0.05 & 0.14 \\
\hline
\end{tabular}

$T_{\mathrm{m}}=$ temperature at which maximum occurs; $T_{v}=$ glass transition temperature. 
given as (Hunklinger and Arnold 1976)

$$
P(V)=\left[P_{0} / V_{0} \sqrt{ } 2 \pi\right] \exp \left[-\left(V-V_{m}\right)^{2} / 2 V_{0}\right],
$$

where $P_{0}=\int_{0}^{\infty} P(V) \mathrm{d} V, 2 V_{0}=$ width at half maximum and $V_{m}$ is the centre. A similar expression has also been used (Berret et al 1985) to fit ultrasonic data on another glass (La SF7). However, the physical origins of these parameters are not clear. Recently Birge et al (1984) used an expression of the type given below to explain $\beta$ relaxation in $(\mathrm{KBr})_{x}(\mathrm{KCN})_{1-x}$

$$
P(V)=\left[1 /(\pi \sigma)^{1 / 2}\right] \exp \left[\left(V-V_{m}\right)^{2} / \sigma^{2}\right],
$$

where $\sigma$ is the temperature dependent width.

A recent theory of $(\mathrm{KBr})_{0.5}(\mathrm{KCN})_{0.5}$ explains the physical meaning of the parameters in (2). (Sethna et al 1984; Meissner et al 1985). We have investigated the available data of dielectric measurements of $\beta$-relaxation and found an expression of the type (2) can fit most of the data (Rajeev and Raychaudhuri 1986). We are now trying to correlate these fit parameters with physical quantities as a first step to make a model for $\beta$-relaxation.

\section{TLS-relaxation}

In 1971 it was observed that thermal properties of glasses are substantially different from their crystalline counter parts (Zeller and Pohl 1971). Below $1 \mathrm{~K}$, the specific heat varies almost linearly with $T$ whereas in crystals it show a Debye $T^{3}$ law (see figure 3). The thermal conductivity also shows a quadratic temperature dependence (see figure 4). This behaviour is now found to be universal to all glasses. We refer to two review articles for a detailed discussion (Phillips 1981; Hunklinger and Raychaudhuri 1986).

It is now generally believed that this excess specific heat and low thermal conductivity

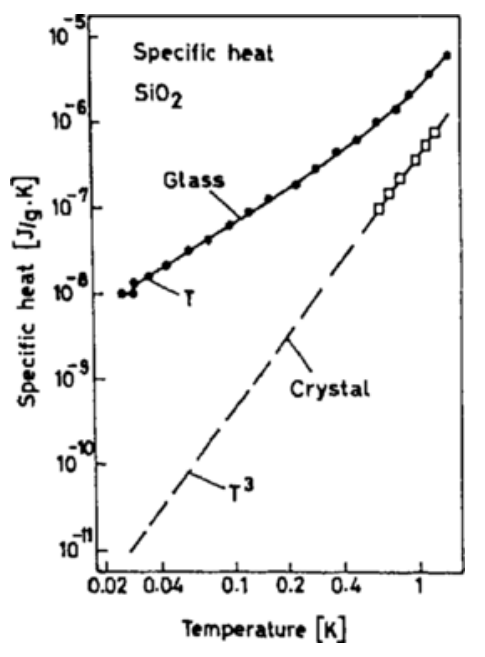

Figure 3. Specific heats of vitreous silica and crystalline quartz at low temperature (Zeller and Pohl 1971). 


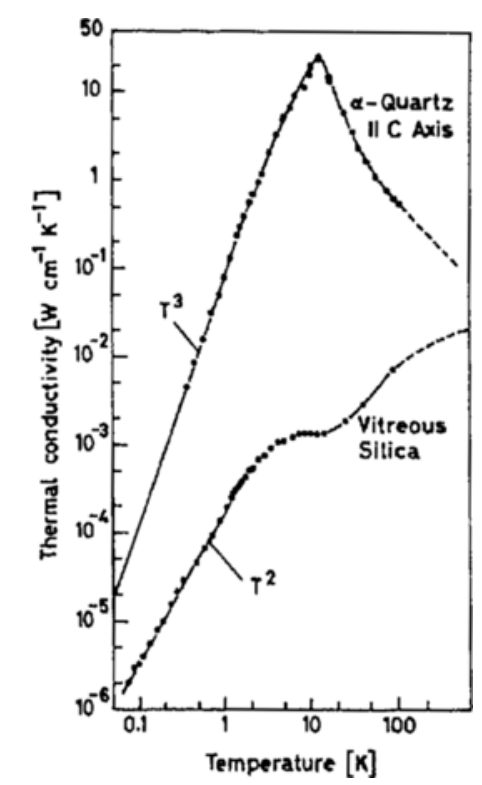

Figure 4. Thermal conductivities of vitreous silica and crystalline quartz (Zeller and Pohl 1971).

of glass is caused by excess excitations which are like two level systems (TLS). The TLs is believed to arise from tunnelling (quantum mechanical) of atoms or molecules (Anderson et al 1972; Phillips 1972). However, not much is known about the tunnelling units in actual glasses.

The low temperature acoustic and dielectric behaviour of glasses are mainly controlled by relaxation of these TLS. The TLS can also resonantly interact with lattice vibration (phonons) giving rise to the quadratic thermal conductivity. The references mentioned above discuss in detail all these aspects. In this paper we will point out what we think are the important parameters in TLS relaxation and the recent attempts to correlate them to bulk material properties. In the tunnelling model the two most important parameters are $\bar{P}$ the density of states of TLS and the deformation potentials for TLS-lattice interaction. At present these two have to be obtained from experiments (Hunklinger and Raychaudhuri 1985). The task is now first to obtain at least empirical correlation of $\bar{P}$ and $y$ with other properties like glass transition temperature $\left(T_{\theta}\right)$, sound velocity etc. From this correlation a model of tunnelling units can then be made. The first attempt in that direction has already started. The earliest correlation to be observed was between $l$ (the phonon mean free path, which contains both $\bar{P}$ and $\gamma$ ) and $T_{g}$ (Reynolds 1979). Later a correlation was found between $P_{0}$ and $T_{g}$ (Raychaudhuri and Pohl 1981, 1982; Doussineau et al 1983). Recent studies (MacDonald et al 1985) support the correlation of $l$ and $T_{g}$. Recently these measurements have been extended to glass forming systems with lower $T_{\theta}$ (Reichert et al 1985) and in 21 glasses it has been found that a relation of the following type holds.

$$
P=\bar{P}_{0} \exp \left(\Delta / k T_{\theta}\right)
$$

where $\bar{P}_{0}=5.5 \times 10^{44} / \mathrm{J} \cdot \mathrm{m}^{3}$ and $\Delta / k=630 \mathrm{~K}$. The glasses for which the above 
relation holds have $T_{g}$ lying between $135 \mathrm{~K}$ and $1500 \mathrm{~K}$. It can be seen that for glasses with $k T_{\theta} \approx \Delta, \bar{P} \propto T_{a}^{-1}$. Equation (3) is indeed a remarkable one. And in our opinion will be the starting point for many a theory and model in future.

The correlation of $\gamma$ with other properties are not yet very successful. But it seems that in samples with low $T_{g}, \gamma \propto T_{q}$.

Much work is required in this field before a proper structure property correlation can be attempted.

\section{Conclusion}

The above discussion is intended to highlight the problems of low energy relaxation in glasses. As it can be seen there is scope for much work in this field. In our opinion to gain an understanding the attempt should be directed towards a structure propertycorrelation. In this paper we have shown that the first stage of identifying the relevant relaxation parameters and establishing an empirical correlation with other bulk properties is already on its way. In the next stage, even a simple model to explain these properties in a particular glass will be a great step forward.

\section{References}

Anderson P W, Halperin B I and Varma C M 1972 Philos. Mag. 251

Barmatz M and Chen H S 1974 Phys. Rev. 94073

Berret J F, Vacher R, Raychaudhuri A K and Schemidt M 1985 J. Non-Cryst. Solids (to be published)

Birge N, Geong Y H and Nagel S R, Bhattacharya S and Susman S 1984 Phys. Rev. B30 2306

Doussineau P, Mateckii M and Schon W 1983 J. Phys. (Paris) 44101

Hunklinger S and Arnold W A 1976 Physical Acoustics (eds) R N Thwiston and W P Mason (New York: Academic Press) Vol 12

Hunklinger S and Raychaudhuri A K 1986 Progress in low temperature physics (Amsterdam: North Holland)

Johari G P 1976 Ann. N.Y. Acad. Sci. 279117

MacDonald W M, Anderson A C and Schroeder J 1985 Phys. Rev. B31 1090

Meissner M, Knaakk W, Sethna J P, Chow K S, Deyoreo J and PohJ R O 1985 Phys. Rev. B32 6091

Philips W A 1972 J. Low Temp. Phys. 7351

Phillips W A 1981 Amorphous solids--low temperature properties (Berlin: Springer)

Rajoev K P and Raychaudhuri A K 1986 J. Non-Cryst. Solids (submitted)

Rao K J and Parthasarathy R 1985 Proc. Indian Acad. Sci. (Chem. Sci.) 94201.

Raychaudhuri A K and Hunklinger S 1984 Z. Phys. B75 113

Raychaudhuri A K and Pohl R O 1981 Solid State Commun. 37105

Raychaudhuri A K and Pohl R O 1982 Phys. Rev. B25 1310

Raychaudhuri A K 1986 Phys. Rev. (submitted)

Reichert U, Schmidt M and Hunklinger S 1985 Solid State Commun. (to be published)

Reynolds Jr. C L 1979 J. Non-Cryst. Solids 30371

Sethna J P, Nagel S R and Ramakrishnan T V 1984 Phys. Rev. Lett. 532489

Silvertsen M 1953 J. Appl. Phys. 2481

Zeller R C and Pohl R O 1971 Phys. Rev. B4 2029 